\title{
Solid-state NMR Paramagnetic Relaxation Enhancement Immersion Depth Studies in Phospholipid Bilayers
}

\author{
Shidong Chu, Sergey Maltsev, A-H Emwas, and Gary A. Lorigan ${ }^{\star}$ \\ Department of Chemistry and Biochemistry, Miami University, Oxford, OH, USA 45056
}

\begin{abstract}
A new approach for determining the membrane immersion depth of a spin-labeled probe has been developed using paramagnetic relaxation enhancement (PRE) in solid-state NMR spectroscopy. A DOXYL spin label was placed at different sites of 1-palmitoyl-2-stearoyl-sn-glycero-3phosphocholine (PSPC) phospholipid bilayers as paramagnetic moieties and the resulting enhancements of the longitudinal relaxation $\left(\mathrm{T}_{1}\right)$ times of ${ }^{31} \mathrm{P}$ nuclei on the surface of the bilayers were measured by a standard inversion recovery pulse sequence. The ${ }^{31} \mathrm{P}$ NMR spin-lattice relaxation times decrease steadily as the DOXYL spin label moves closer to the surface as well as the concentration of the spin-labeled lipids increase. The enhanced relaxation $v s$. the position and concentration of spin-labels indicate that PRE induced by the DOXYL spin label are significant to determine longer distances over the whole range of the membrane depths. When these data were combined with estimated correlation times $\tau_{\mathrm{c}}$, the $r^{-6}$-weighted, time-averaged distances between the spin labels and the ${ }^{31} \mathrm{P}$ nuclei on the membrane surface were estimated. The application of using this solid-state NMR PRE approach coupled with site-directed spin labeling (SDSL) may be a powerful method for measuring membrane protein immersion depth.
\end{abstract}

\section{Keywords}

solid-state NMR; paramagnetic relaxation enhancement; phospholipid membranes; spin-lattice relaxation

\section{Introduction}

One important piece of structural information of integral membrane proteins is the immersion depth within membrane, i.e., how far a specific site of the protein is located from the membrane surface. Knowledge of the precise depth of a specific site or residue is essential in defining the overall topology and conformation of the proteins in the membranes, and helps in understanding the function and action mechanism of the system under investigation [1-5]. This is especially true since the full three-dimensional structures are not easily available for membrane proteins and macromolecular complexes.

A variety of biophysical techniques, with different intrinsic advantages and disadvantages, have been developed and employed to study the immersion depth of membrane proteins. Fluorescence techniques [6-10], FT-IR [11], and especially electron paramagnetic resonance

\footnotetext{
*Corresponding author: Dr. Gary A. Lorigan, Tel: +1 513529 3338, Fax: +1 513529 5715. garylorigan@ muohio.edu.

Publisher's Disclaimer: This is a PDF file of an unedited manuscript that has been accepted for publication. As a service to our customers we are providing this early version of the manuscript. The manuscript will undergo copyediting, typesetting, and review of the resulting proof before it is published in its final citable form. Please note that during the production process errors may be discovered which could affect the content, and all legal disclaimers that apply to the journal pertain.
} 
(EPR) spectroscopy [12-21], including power saturation [17], ESEEM [19,20], have been widely used to obtain the immersion depth due to their relatively high sensitivity. The precision in determining the insertion depth using fluorescence and EPR spectroscopic methods is about $1.5-3 \AA$, owing to the size of either fluorescent or spin probes attached. Scattering techniques, mainly x-ray [22] and neutron diffraction [23,24], can yield a resolution of $\sim 0.2 \AA$. A crucial issue for neutron diffraction is the detection limit for deuterium (i.e., the number of deuterons per lipid that can be detected) [24]. While most of the above techniques require sample labeling, deuteration for neutron diffraction, fluorophore-labeling for fluorescence quenching, or spinlabeling for EPR spectroscopy, NMR spectroscopy, either in the liquid-state [25-29] or in the solid-state [3,5,30-34], do not necessarily require isotopic labeling as long as reasonable sensitivity is achieved.

In standard solution NMR spectroscopy, the range of distance measured by NOE-based experiments is relatively short $(\sim 5 \AA)$, owing to the weak interaction between nuclear dipolar magnetic moments. This interaction can be enhanced significantly by replacing the nuclear dipolar coupling with the much stronger electron-nuclear dipolar interaction, thus, resulting in paramagnetic relaxation enhancement (PRE) [35]. Previous theoretical and experimental studies have shown that significant PRE is expected for distances of 5-30 $[35,45]$. PRE can serve as an ideal technique for determining the penetration depth in the membrane, since the thickness of a lipid bilayer is approximately $~ 30-50 \AA$.

Paramagnetic relaxation enhancement has been used in solution NMR spectroscopy to determine the immersion depth $[25-28,36]$ and distances between paramagnetic centers [37, 38]. The paramagnetic agents can either be covalently linked to the site of interest $[39,40]$ or freely soluble in the buffer surrounding the membrane environment, e.g. $\mathrm{Mn}^{2+}[41,42], \mathrm{O}_{2}$ [43], lanthanide ions [44], or chelated paramagnetic metals [25,28]. One of the potential problems with free movable paramagnetic agents is the interaction between the agents and the proteins, which may lead to re-organization of the proteins in the membranes and change the native topology and conformation of the membrane proteins. Conversely, while PRE has been explored in solution NMR [45], it has been underutilized in solid-state NMR spectroscopy, whereas PRE is more pronounced in solid-state than in liquids [46]. Since solid-state NMR spectroscopy can provide powerful structural and dynamic information for investigating proteins in membranes, it is thus desirable to explore more fully the intrinsic benefits of PRE with solid-state NMR [41,46-50].

One of our long-term research goals is to fully explore the unique advantages of both solidstate NMR and EPR spectroscopy and to develop new structural biology tools for studying membrane proteins [51-53]. Since ${ }^{31} \mathrm{P}$ nuclei are abundant at the surface of phospholipid membranes and are very NMR sensitive, we reasoned that site-directed spin-labeled (SDSL) proteins might be ideally used to determine the distances between the ${ }^{31} \mathrm{P}$ nuclei and the spin labels by ${ }^{31} \mathrm{P}$ solid-state NMR PRE, and one such application would be the determination of membrane insertion depth. In the work presented here, we employed a series of doxyl labeled 1-palmitoyl-2-stearoyl-sn-glycero-3-phosphocholine (n-DOXYL-PSPC, where $\mathrm{n}=5,10,14$, Fig. 1A) embedded into PSPC phospholipid bilayers. ${ }^{31} \mathrm{P}$ longitudinal relaxation enhancement was determined to obtain qualitative measurements of the immersion depth of the spin-labels in the lipid bilayers (Fig. 1B).

\section{Methods}

All phospholipids were purchased from Avanti Polar Lipids, Inc. (Alabaster, AL) and used without further purification. 1-palmitoyl-2-stearoyl-sn-glycero-3-phosphocholine (PSPC), 1palmitoyl-2-(n-doxylstearoyl)-sn-glycero-3-phosphocholine (n-DOXYL-PSPC, where $\mathrm{n}=5$, 10 , or 14) were dissolved in chloroform and stored at $-20 \mathrm{C}^{\circ}$ prior to use. 
Multilamellar vesicles (MLVs) of pure PSPC or PSPC/n-DOXYL-PSPC mixtures in different molar ratios were prepared according to our previously reported procedure [54]. A total amount of $~ 70 \mathrm{mg}$ of PSPC lipids was dissolved in chloroform and appropriate amounts of n-DOXYLPSPC were mixed to obtain different molar ratios of spin-labels. The chloroform solvent was removed slowly under a steady stream of pure $\mathrm{N}_{2}$ gas and the samples were dried overnight in a vacuum desiccator. The resulting mixtures were fully hydrated with $190 \mu \mathrm{L}$ HEPES buffer (30 mM HEPES, $20 \mathrm{mM} \mathrm{NaCl}, \mathrm{pH}$ 7.4). The hydrated samples went through 5-10 freezethawing cycles to increase the homogeneity of the vesicles. The pellet $(60-100 \mathrm{mg})$ was transferred to a $4 \mathrm{~mm}$ MAS NMR rotor for ${ }^{31} \mathrm{P}$ NMR spectroscopic studies.

All NMR data were collected on a Bruker AVANCE $500 \mathrm{MHz}$ wide-bore NMR spectrometer equipped with a CP-MAS probe (Bruker Biospin, Rheinstetten, Germany). ${ }^{31} \mathrm{P}$ longitudinal relaxation times $\left(\mathrm{T}_{1}\right)$ were measured using a standard one-dimensional direct phosphorous detection inversion-recovery pulse sequence $\left(180^{\circ}-\mathrm{T}-90^{\circ}\right.$ - acquire $)$, with a pre-acquisition delay of $10 \mathrm{~s}$, and 14 relaxation delay D1 values ranging from $0.2 \mathrm{~ms}$ to $7 \mathrm{~s}$. The sample was spinning at a frequency of $4 \mathrm{kHz}$ at the magic angle. $\mathrm{T}_{1}$ values were calculated by fitting the ${ }^{31} \mathrm{P}$ MAS isotropic peak intensities to a single exponential function $\mathrm{I}(\mathrm{t})=\mathrm{I}(0)+\mathrm{P}^{*} \exp (-\mathrm{t} /$ $\mathrm{T}_{1}$ ) using Topspin 2.0 (Bruker Biospin).

The paramagnetic relaxation enhancement (PRE, described as $\Gamma_{1}$ in the following sections) is determined by subtracting the longitudinal relaxation rate of the membrane system in the absence of paramagnetic labeling $\left(\mathrm{R}_{1 \text { (dia) }}\right.$ or $\left.1 / \mathrm{T}_{1 \text { (dia) }}\right)$ from the relaxation rate in the presence of spin-labeled species ( $\mathrm{R}_{1 \text { (para) }}$, or $\left.1 / \mathrm{T}_{1 \text { (para) }}\right)$ according to Eq. 1

$$
\Gamma_{1}=R_{1(\text { para })}-R_{1(\text { dia })=} \frac{1}{T_{1(\text { para })}}-\frac{1}{T_{1(\text { dia })}}
$$

PRE $\Gamma_{1}$ can be calculated according to the modified Solomon-Bloembergen equations (Eq. 2) $[37,45]$.

$$
\Gamma_{1}=\frac{2}{5}\left(\frac{\mu_{0}}{4 \pi}\right)^{2} \gamma_{1}^{2} g^{2} \mu_{B}^{2} S(S+1) J_{S B}\left(\omega_{r}\right)
$$

Where $\gamma_{r}$ is the gyromagnetic ratio of ${ }^{31} \mathrm{P}, g$ is the electron $\mathrm{g}$-factor, $\mu_{0}$ is the magnetic permeability of vacuum, $\mu_{B}$ is the electron Bohr magneton, $S$ is the electron spin quantum number, $\omega_{I} / 2 \pi$ is the Larmor precession frequency of ${ }^{31} \mathrm{P}, \mathrm{J}_{\mathrm{SB}}\left(\omega_{\mathrm{r}}\right)$ is the generalized spectral density for the reduced correlation function, which is a Lorentzian function of $\omega$ :

$$
J_{S B}\left(\omega_{r}\right)=r^{-6} \frac{\tau_{c}}{1+\left(\omega \tau_{c}\right)^{2}}
$$

Where $r$ is the distance between the unpaired electron (approximately localized on the nitrogen atom of the nitroxide DOXYL spin label) and the observed nuclear spins (here the ${ }^{31} \mathrm{P}$ nucleus), $\tau_{\mathrm{c}}$ is the correlation time of the electron $-{ }^{31} \mathrm{P}$ dipolar interaction. The general dependence of the distance $r$ between the spin label and the ${ }^{31} \mathrm{P}$ nucleus on the correlation time is shown on Fig. 2. For the distance calculations an approximation was made that $\tau_{c}$ was put to be equal to the global correlation time of the spin-labeled lipids.

In this paper, the items $\frac{2}{5}\left(\frac{\mu_{0}}{4 \pi}\right)^{2} \gamma_{I}^{2} g^{2} \mu_{B}^{2} S(S+1)$ are combined into a constant $K$. For ${ }^{31} \mathrm{P}$ nuclei,
$K=1.21 \times 10^{-32} \mathrm{~cm}^{6} \mathrm{~s}^{-2}$ 
Thus, Eq. 2 can be re-written as:

$$
r=\left[\frac{K}{\Gamma_{1}} \frac{\tau_{c}}{1+\left(\omega \tau_{c}\right)^{2}}\right]^{1 / 6}
$$

Now, the DOXYL concentration should be taken into account. This can be done by integrating ${ }^{31} \mathrm{P}$ relaxation signals from all ${ }^{31} \mathrm{P}$ atoms of the lipid surface. The integration area can be estimated as proportional to the square root of the DOXYL-labeled lipids/overall lipids ratio (i.e. the DOXYL-label concentration). Thus the inversion recovery signal can be described as follows:

$$
S=\int_{0}^{R \lim } 2 \pi r \rho\left(y_{o}+A \exp \left(-\frac{t}{T p s p c}-\frac{K}{\left(r^{2}+d^{2}\right)^{3}} \frac{t \tau_{C}}{1+\left(\omega \tau_{C}\right)^{2}}\right)\right) d r
$$

where $\rho$ is DOXYL concentration, $y_{0}$ and $A$ are the level offset and the amplitude of the measured inversion recovery curve, Tpspc is the relaxation time of pure PSPC lipids, $r, d$ and $R_{l i m}$ are defined as in Fig. 5. This equation has an analytical solution:

$$
\begin{aligned}
& S=\pi\left(R_{\text {lim }}{ }^{2}+d^{2}\right) \rho\left(y_{o}+A \exp \left(-\frac{t}{T p s p c}-\frac{K}{\left(R_{\lim }{ }^{2}+d^{2}\right)^{3}} \frac{t \tau_{C}}{1+\left(\omega \tau_{C}\right)^{2}}\right)-\right. \\
& \left.-A \exp \left(-\frac{t}{T p s p c}\left(\frac{K}{\left(R_{\text {lim }}{ }^{2}+d^{2}\right)^{3}} \frac{t \tau_{C}}{1+\left(\omega \tau_{C}\right)^{2}}\right)^{\frac{1}{3}}\right) \Gamma\left(\frac{2}{3}, \frac{K}{\left(R_{\lim }{ }^{2}+d^{2}\right)^{3}} \frac{t \tau_{C}}{1+\left(\omega \tau_{C}\right)^{2}}\right)\right)
\end{aligned}
$$

where $\Gamma$ is upper incomplete gamma function. Fitting this equation to the experimental data gives a relaxation behavior that accounts for ${ }^{31} \mathrm{P}-$ DOXYL-label distance distribution and allows a more accurate calculation of the DOXYL depth. Further we refer to the relaxation behavior described by (6) as "geometry weighted relaxation".

\section{Results}

PSPC vesicles with spin-label located at three different depths (Fig. 1) of the lipid bilayer was added respectively to the control pure PSPC lipid bilayer and the ${ }^{31} \mathrm{P} \mathrm{T}_{1}$ time values were determined using the inversion-recovery pulse sequence. The inversion recovery data and the corresponding $\mathrm{T}_{1}$ fits for the $\mathrm{n}$-DOXYL-PSPC/PSPC $(\mathrm{n}-5,10,14)$ along with that for the pure PSPC lipid vesicles are shown in Fig. 3. In all cases, the decay resulting from the relaxation phenomenon can be fitted satisfactorily with a single exponential behavior, suggesting that there is a single predominant contribution to the relaxation process. However, fitting with geometry weighted relaxation model gives slightly different values. As clearly observed in Fig. 3 , adding the paramagnetic agents of $n$-DOXYL PSPC lipids induced faster ${ }^{31} \mathrm{P} \mathrm{T}_{1}$ relaxation. As expected the relaxation enhancement depends upon the position of the DOXYL spin label with respect to the ${ }^{31} \mathrm{P}$ nuclei on the membrane surface.

The ${ }^{31} \mathrm{P} \mathrm{T}_{1}$ values for the systems studied are summarized in Table 1 . To address the paramagnetic relaxation enhancement of the nuclear spin-lattice relaxation rates that results from intermolecular magnetic dipole-dipole interactions, and to find the optimal concentration of spin-labels to lipid, a titration of the 5-DOXYL-PSPC vesicles was conducted, and the corresponding ${ }^{31} \mathrm{PT}_{1}$ relaxation times were measured. Plotting $\mathrm{T}_{1}$ relaxation times as a function of the molar ratio of the spin-labels yields a straight line whose slope is the paramagnetic 
relaxation enhancement (Fig. 4). As expected, the $T_{1}$ values depend linearly on the relative molar fraction of the spin-label concentration in MLVs [56].

We evaluated the contribution of the intermolecular magnetic dipole-dipole interactions to the PRE at low concentration of paramagnetic species by estimating the distance between the ${ }^{31} \mathrm{P}$ nuclei and the nearby spin label (Fig. 5). The surface area of per PSPC molecule is $67 \AA^{2}$ [57] which results in a separation of $\mathrm{R}=8.2 \AA$ between the two head groups. At the concentration of 5\% mol n-DOXYL PSPC, the next nearest spin label will be about $37 \AA$ Away from the ${ }^{31} \mathrm{P}$ nucleus (Fig. 5) and thus, due to the strong distance dependence, the PRE effect can be seen as a single nucleus-spin label process for the distances of interest in the range of 10-17 ̊̊. It should be noted that in general one does not need to carry out a full titration with spin-labels. Instead, acquiring relaxation times in the absence and presence of a significant concentration of the spin-labeled samples should suffice.

\section{Discussion}

The membrane immersion depth, or penetration depth, is not well defined in the literature. In fluorescence studies developed by London [6], often the distance from the spin-label to the bilayer center was estimated. In EPR spectroscopy studies, generally another paramagnetic agent needs to be added to measure the distance between the spins and hence the immersion depth. In a neutron diffraction study [24], distance of deuterated amino acids from the bilayer center were determined. In ESEEM spectroscopy, the head groups of lipids were deuterated and the distance between deuterium and the spin, which is placed at different positions in the membrane, were determined [20]. In previous NMR PRE studies in micelles, the distance between the nucleus and paramagnetic agents such as $\mathrm{Mn}^{2+}$ on the surface of lipid bilayer [42], chelated paramagnetic metal Gd(DTPA-BMA) on the surface of the micelle $[25,28,56]$ were used.

${ }^{31} \mathrm{P}$, with a $100 \%$ natural abundance and an intermediate gyromagnetic ratio, are distributed uniformly on the surface of the lipid bilayer and can be used as an ideal reference for immersion depth measurements by NMR spectroscopy. The marked enhancement of the relaxation efficiency observed in the presence of the paramagnetic species (Fig. 3, Fig. 4 and Table 1) is the result of the magnetic moment of the unpaired electron's being almost 1000 times higher than that of the ${ }^{31} \mathrm{P}$ nucleus. The dipolar interaction between the electron and nuclear spins is much stronger than the dipolar interaction between two nuclei, and its strength depends on the distance and is not influenced by the medium in between them; therefore, such measurements are potentially very precise. This approach also eliminates the necessity for addition of paramagnetic agents on the surface of the membrane or deuteration of the headgroups of the lipids.

The paramagnetic DOXYL probe used in this paper is a nitroxyl ring attached rigidly at a specific carbon position on the chain of PSPC lipids. Previous studies have shown that DOXYL-PSPC is rather non-perturbing for lipid membranes [58].

As shown in Fig 3, introduction of the spin-labels induced significant PRE effect as observed in the changes of the ${ }^{31} \mathrm{P} \mathrm{T}_{1}$ times and the resulting PRE show clear dependence on the distances between the spin labels and the ${ }^{31} \mathrm{P}$ nuclei. This PRE provides a relative spectroscopic ruler to measure the depth of a specific site in the membranes. PRE also depends upon the concentration of the spin labels (Fig. 4) and the correlation time $\tau_{c}$. The higher concentration of spin labels, the more significant PRE is. The graph is linear over the concentration range shown in Figure 4. At higher concentrations, this graph may not be linear because of lipid demixing. Our single pulse ${ }^{31} \mathrm{P}$ NMR data indicate that $5 \%$ mol of spin labels is enough for inducing a significant PRE, while keeping the structure of the membrane intact. 
The estimated PRE distances in Table 1 were compared with the available literature distances [12]. The exact distance between ${ }^{31} \mathrm{P}$ and spin labeled lipid membranes is not available in the literature. According to force field and density functional theory (DFT) calculations, expected distances for an all-trans conformation of the hydrocarbon chain are $10 \AA$ for 5-DOXYL-PSPC in the gas phase [59]. By using fluorescence quenching, the distances from the hydrophobic center of the bilayers to the spin-label were estimated to be $12.2 \AA$ for the 5-DOXYL PSPC [6], which gives a distance of $\sim 11 \AA$ between the 5-DOXYL and the membrane surface, assuming the thickness of a PSPC bilayer to be $46 \AA$ [60]. Our corresponding calculated distance of $10.6 \AA$ for 5-DOXYL-PSPC is in a reasonable range.

When the spin-label is moving towards the center of the PSPC bilayer, ${ }^{31} \mathrm{P} \mathrm{T}_{1}$ relaxation times and corresponding distances increase (see Table 1). It has been shown that correlation times vary with the positions in DOXYL-labeled stearic acid (6.5 ns for 5-SDA, $12.4 \mathrm{~ns}$ for 10-SDA) [61]. We used an estimated correlation time $\tau_{\mathrm{c}}$ in calculating the distance from the PRE data of $1 \mathrm{~ns}$. Because the distance depends on the sixth-root of the correlation time, the error in distance $\boldsymbol{r}$ introduced by even large errors in correlation time $\tau_{\mathrm{c}}$ is relatively small. For instance, a relative error of $50 \%$ in the correlation time for a given site would produce an error of about $9 \%$ in the measured distance, or approximately $\pm 1.5 \AA$ for a distance of $15 \AA$ [55]. The calculation yields the following values for the ${ }^{31} \mathrm{P}$-spin label distances: $12.6,13.3$ and $16.6 \AA$, for 5-, 10- and 14-DOXYL PSPC respectively for a single exponential fit and 10.6, 12.3 and 16.0 for a geometry weighted relaxation fit which are more physically appropriate. This difference clearly shows the necessity of taking into account the relaxation signals from all nearby lipids.

An obvious improvement of this approach is to conveniently determine the correlation time with EPR spectroscopy [62]. Another source of error is the relaxation curves fitting procedure. Since $\Gamma_{1}$ is in the denominator and is a difference between two relaxation rates a careful estimation of relaxation times is needed especially for the long distances as a small error in $\Gamma_{1}$ calculation can lead to huge errors in distance calculations.

Several assumptions were made in our model of membrane immersion depth determination by ${ }^{31} \mathrm{P} \mathrm{PRE} \mathrm{T}_{1}$ of the phospholipids with spin labeling. The electron- ${ }^{31} \mathrm{P}$ distance in fact is the most probable distance of a paramagnetic center to the planar surface of phospholipid membranes because the ${ }^{31} \mathrm{P}$ nuclei are distributed uniformly in a plane of lipid membranes. It includes distance averaging due to molecular dynamics, lipid chain tilt angles, etc. In membrane peptide/protein systems, the molecular dynamics of the lipid will be much less significant, which should lead to an even more accurate measurement of immersion depth of proteins/ peptides with angstrom resolution. A more detailed theory of PRE in lipids is being prepared in our group that will account for spin label concentration, different correlation time for each spin-label position and molecular dynamics.

With all limitations described above, PRE-based insertion depth measurements have obviously many advantages and when compared to other techniques. There is no need to have high-quality crystals and no limitation on molar mass. In EPR spectroscopy, another paramagnetic agent needs to be added to measure the distance between the spins and hence the immersion depth. The experiment can be conducted in the absence of any protein, in the presence of spin labeled protein, or proteins without spin labeling, and thus determine if the protein position and the overall structure of the bilayers changes in the presence of the label. The solid-state NMR method has the advantage of not requiring the coupling of an extrinsic spin probe to the protein.

In summary, we provided a qualitative estimation of distances between paramagnetic centers embedded in lipid bilayers and the ${ }^{31} \mathrm{P}$ nuclei membrane surface and evaluated its feasibility in determining the immersion depth. Often for large macromolecular complexes in the 
membrane, a few critical structural features including the location of a specific site can provide many insights on the function and mechanism of action. Combined with EPR spectroscopy this technique looks very promising in providing site-specific distance determination in complex systems where it is very challenging for many other techniques. We are extending the methodology developed here to determine the immersion depth of membrane proteins via SDSL coupled with ${ }^{31} \mathrm{P}$ MAS solid-state NMR spectroscopy.

\section{Abbreviations used}

$\begin{array}{ll}\text { PSPC } & \text { 1-palmitoyl-2-stearoyl-sn-glycero-3-phosphocholine } \\ \text { n-DOXYL-PSPC } & \text { 1-palmitoyl-2-stearoyl-(n-doxyl)-sn-glycero-3-phosphocholine } \\ \text { ESEEM } & \text { Electron Spin Echo Envelope Modulation } \\ \text { MAS } & \text { Magic-Angle Spinning } \\ \text { PRE } & \text { paramagnetic relaxation enhancement }\end{array}$

\section{Acknowledgments}

This work was partially supported by the NIH grant GM080542. The Bruker $500 \mathrm{MHz}$ wide bore NMR spectrometer was obtained from the NSF grant 10116333.

\section{References}

1. Shai Y. Mechanism of the binding, insertion and destabilization of phospholipid bilayer membranes by alpha-helical antimicrobial and cell non-selective membrane-lytic peptides. Biochim. Biophys. Acta-Biomembr 1999;1462:55-70.

2. Popot JL, Engelman DM. Helical membrane protein folding, stability, and evolution. Annu. Rev. Biochem 2000;69:881-922. [PubMed: 10966478]

3. Hong M. Structure, topology, and dynamics of membrane peptides and proteins from solid-state NMR Spectroscopy. J. Phys. Chem. B 2007;111:10340-10351. [PubMed: 17685648]

4. Bechinger B. Rationalizing the membrane interactions of cationic amphipathic antimicrobial peptides by their molecular shape. Curr. Opin. Colloid Interface Sci 2009;14:349-355.

5. Qiang W, Sun Y, Weliky DP. A strong correlation between fusogenicity and membrane insertion depth of the HIV fusion peptide. Proc. Natl. Acad. Sci. U.S.A 2009;106:15314-15319. [PubMed: 19706388]

6. Chattopadhyay A, London E. Parallax method for direct measurement of membrane penetration depth utilizing fluorescence quenching by spin-labeled phospholipids. Biochemistry 1987;26:39-45. [PubMed: 3030403]

7. Clague MJ, Knutson JR, Blumenthal R, Herrmann A. Interaction of influenza hemagglutinin aminoterminal peptide with phospholipid-vesicles - a fluorescence study. Biochemistry 1991;30:5491-5497. [PubMed: 2036418]

8. Strashnikova NV, Medvedeva N, Likhtenshtein GI. Depth of immersion of fluorescent chromophores in biomembranes studied by quenching with nitroxide radical. J. Biochem. Biophys. Methods 2001;48:43-60. [PubMed: 11282401]

9. Ramachandran R, Heuck AP, Tweten RK, Johnson AE. Structural insights into the membraneanchoring mechanism of a cholesterol-dependent cytolysin. Nat. Struct. Biol 2002;9:823-827. [PubMed: 12368903]

10. Posokhov YO, Ladokhin AS. Lifetime fluorescence method for determining membrane topology of proteins. Anal. Biochem 2006;348:87-93. [PubMed: 16298322]

11. Huang WN, Sue SC, Wang DS, Wu PL, Wu WG. Peripheral binding mode and penetration depth of cobra cardiotoxin on phospholipid membranes as studied by a combined FTIR and computer simulation approach. Biochemistry 2003;42:7457-7466. [PubMed: 12809502] 
12. Dalton LA, McIntyre JO, Fleischer S. DIstance estimate of the active-center of d-betahydroxybutyrate dehydrogenase from the membrane-surface. Biochemistry 1987;26:2117-2130. [PubMed: 3040081]

13. Altenbach C, Greenhalgh DA, Khorana HG, Hubbell WL. A collision gradient-method to determine the immersion depth of nitroxides in lipid bilayers - application to spin-labeled mutants of bacteriorhodopsin. Proc. Natl. Acad. Sci. U.S.A 1994;91:1667-1671. [PubMed: 8127863]

14. Yu YG, Thorgeirsson TE, Shin YK. Topology of an amphiphilic mitochondrial signal sequence in the membrane-inserted state - a spin-labeling study. Biochemistry 1994;33:14221-14226. [PubMed: 7947833]

15. Monaco V, Formaggio F, Crisma M, Toniolo C, Hanson P, Millhauser GL. Orientation and immersion depth of a helical lipopeptaibol in membranes using TOAC as an ESR probe. Biopolymers 1999;50:239-253. [PubMed: 10397787]

16. Bhargava K, Feix JB. Membrane binding, structure, and localization of cecropin-mellitin hybrid peptides: A site-directed spin-labeling study. Biophys. J 2004;86:329-336. [PubMed: 14695274]

17. Malmberg NJ, Falke JJ. Use of EPR power saturation toanalyze the membrane-docking geometries of peripheral proteins: A applications to C2 domains. Annu. Rev. Biophys. Biomol. Struct 2005;34:71-90. [PubMed: 15869384]

18. Nielsen RD, Che KP, Gelb MH, Robinson BH. A ruler for determining the position of proteins in membranes. J. Am. Chem. Soc 2005;127:6430-6442. [PubMed: 15853351]

19. Salnikov ES, Erilov DA, Milov AD, Tsvetkov YD, Peggion C, Formaggio F, Toniolo C, Raap J, Dzuba SA. Location and aggregation of the spin-labeled peptide trichogin GA IV in a phospholipid membrane as revealed by pulsed EPR. Biophys. J 2006;91:1532-1540. [PubMed: 16751238]

20. Carmieli R, Papo N, Zimmermann H, Potapov A, Shai Y, Goldfarb D. Utilizing ESEEM spectroscopy to locate the position of specific regions of membrane-active peptides within model membranes. Biophys. J 2006;90:492-505. [PubMed: 16258052]

21. Jao CC, Hegde BG, Chen J, Haworth IS, Langen R. Structure of membrane-bound alpha-synuclein from site-directed spin labeling and computational refinement. Proc. Natl. Acad. Sci. U.S.A 2008;105:19666-19671. [PubMed: 19066219]

22. Khattari Z, Brotons G, Arbely E, Arkin IT, Metzger TH, Salditt T. SARS E protein in phospholipid bilayers: an anomalous X-ray reflectivity study. Physica B - Condens. Matter 2005;357:34-38.

23. Hristova K, Dempsey CE, White SH. Structure, location, and lipid perturbations of melittin at the membrane interface. Biophys. J 2001;80:801-811. [PubMed: 11159447]

24. Han X, Mihailescu M, Hristova K. Neutron diffraction studies of fluid bilayers with transmembrane proteins: Structural consequences of the achondroplasia mutation. Biophys. J 2006;91:3736-3747. [PubMed: 16950849]

25. Zangger K, Respondek M, Goebl C, Hohlweg W, Rasmussen K, Grampp G, Madl T. Positioning of micelle-bound peptides by paramagnetic relaxation enhancements. J. Phys. Chem. B 2009;113:44004406. [PubMed: 19256533]

26. Cohen Y, Afri M, Frimer AA. NMR-based molecular ruler for determining the depth of intercalants within the lipid bilayer Part II. The preparation of a molecular ruler. Chem. Phys. Lipids 2008;155:114-119. [PubMed: 18691565]

27. Al-Abdul-Wahid MS, Neale C, Pomes R, Prosser RS. A solution NMR approach to the measurement of amphiphile immersion depth and orientation in membrane model systems. J. Am. Chem. Soc 2009;131:6452-6459. [PubMed: 19415935]

28. Franzmann M, Otzen D, Wimmer R. Quantitative use of paramagnetic relaxation enhancements for determining orientations and insertion depths of peptides in micelles. Chembiochem 2009;10:23392347. [PubMed: 19688788]

29. Luchette PA, Prosser RS, Sanders CR. Oxygen as a paramagnetic probe of membrane protein structure by cysteine mutagenesis and 19F NMR spectroscopy. J. Am. Chem. Soc 2002;124:1778-1781. [PubMed: 11853456]

30. Shi L, Traaseth NJ, Verardi R, Cembran A, Gao JL, Veglia G. A refinement protocol to determine structure, topology, and depth of insertion of membrane proteins using hybrid solution and solidstate NMR restraints. J. Biomol. NMR 2009;44:195-205. [PubMed: 19597943] 
31. McDonnell PA, Shon K, Kim Y, Opella SJ. Fd coat protein-structure in membrane environments. J. Mol. Biol 1993;233:447-463. [PubMed: 8411155]

32. Antharam VC, Elliott DW, Mills FD, Farver RS, Sternin E, Long JR. Penetration depth of surfactant peptide KL4 into membranes is determined by fatty acid saturation. Biophys. J 2009;96:4085-4098. [PubMed: 19450480]

33. Henzler-Wildman KA, Martinez GV, Brown MF, Ramamoorthy A. Perturbation of the hydrophobic core of lipid bilayers by the human antimicrobial peptide LL-37. Biochemistry 2004;43:8459-8469. [PubMed: 15222757]

34. Traaseth NJ, Shi L, Verardi R, Mullen DG, Barany G, Veglia G. Structure and topology of monomeric phospholamban in lipid membranes determined by a hybrid solution and solid-state NMR approach. Proc. Natl. Acad. Sci. U.S.A 2009;106:10165-10170. [PubMed: 19509339]

35. Solomon I, Bloembergen N. Nuclear magnetic interactions in the HF molecule. J. Chem. Phys 1956;25:261-266.

36. North CL, Franklin JC, Bryant RG, Cafiso DS. Molecular flexibility demonstrated by paramagnetic enhancements of nuclear-relaxation - application to alamethicin - a voltage-gated peptide channel. Biophys. J 1994;67:1861-1866. [PubMed: 7532020]

37. Iwahara J, Schwieters CD, Clore GM. Ensemble approach for NMR structure refinement against $1 \mathrm{H}$ paramagnetic relaxation enhancement data arising from a flexible paramagnetic group attached to a macromolecule. J. Am. Chem. Soc 2004;126:5879-5896. [PubMed: 15125681]

38. Liang BY, Bushweller JH, Tamm LK. Site-directed parallel spin-labeling and paramagnetic relaxation enhancement in structure determination of membrane proteins by solution NMR spectroscopy. J. Am. Chem. Soc 2006;128:4389-4397. [PubMed: 16569016]

39. Godici PE, Landsber FR. Dynamic structure of lipid-membranes - 13C nuclear magnetic-resonance study using spin labels. Biochemistry 1974;13:362-368. [PubMed: 4358944]

40. Vogel A, Scheidt HA, Huster D. The distribution of lipid attached spin probes in bilayers: Application to membrane protein topology. Biophys. J 2003;85:1691-1701. [PubMed: 12944284]

41. Su Y, Mani R, Hong M. Asymmetric insertion of membrane proteins in lipid bilayers by solid-state NMR paramagnetic relaxation enhancement: A cell-penetrating peptide example. J. Am. Chem. Soc 2008;130:8856-8864. [PubMed: 18597439]

42. Buffy JJ, Hong T, Yamaguchi S, Waring AJ, Lehrer RI, Hong M. Solid-state NMR investigation of the depth of insertion of protegrin-1 in lipid bilayers using paramagnetic Mn2+ Biophys. J 2003;85:2363-2373. [PubMed: 14507700]

43. Prosser RS, Luchette PA, Westerman PW. Using O2 to probe membrane immersion depth by $19 \mathrm{~F}$ NMR. Proc. Natl. Acad. Sci. U.S.A 2000;97:9967-9971. [PubMed: 10954744]

44. Grobner G, Glaubitz C, Watts A. Probing membrane surfaces and the location of membraneembedded peptides by 13C MAS NMR using lanthanide ions. J. Magn. Reson 1999;141:335-339. [PubMed: 10579957]

45. Clore GM, Iwahara J. Theory, Practice, and applications of paramagnetic relaxation enhancement for the characterization of transient low-population states of biological macromolecules and their complexes. Chem. Rev 2009;109:4108-4139. [PubMed: 19522502]

46. Nadaud PS, Helmus JJ, Hofer N, Jaroniec CP. Long-range structural restraints in spin-labeled proteins probed by solid-state nuclear magnetic resonance spectroscopy. J. Am. Chem. Soc 2007;129:75027503. [PubMed: 17530852]

47. Nadaud PS, Helmus JJ, Kall SL, Jaroniec CP. Paramagnetic ions enable tuning of nuclear relaxation rates and provide long-range structural restraints in solid-state nmr of proteins. J. Am. Chem. Soc 2009;131:8108-8120. [PubMed: 19445506]

48. Wickramasinghe NP, Kotecha M, Samoson A, Past J, Ishii Y. Sensitivity enhancement in 13C solidstate NMR of protein microcrystals by use of paramagnetic metal ions for optimizing $1 \mathrm{H} \mathrm{T1}$ relaxation. J. Magn. Reson 2007;184:350-356. [PubMed: 17126048]

49. Linser R, Chevelkov V, Diehl A, Reif B. Sensitivity enhancement using paramagnetic relaxation in MAS solid-state NMR of perdeuterated proteins. J. Magn. Reson 2007;189:209-216. [PubMed: 17923428] 
50. Thomas L, Scheidt HA, Bettio A, Beck-Sickinger AG, Huster D, Zschornig O. The interaction of neuropeptide $\mathrm{Y}$ with negatively charged and zwitterionic phospholipid membranes. Eur. Biophys. J. Biophys. Lett 2009;38:663-677.

51. Inbaraj JJ, Cardon TB, Laryukhin M, Grosser SM, Lorigan GA. Determining the topology of integral membrane peptides using EPR spectroscopy. J. Am. Chem. Soc 2006;128:9549-9554. [PubMed: 16848493]

52. Inbaraj JJ, Laryukhin M, Lorigan GA. Determining the helical tilt angle of a transmembrane helix in mechanically aligned lipid bilayers using EPR spectroscopy. J. Am. Chem. Soc 2007;129:7710 7711. [PubMed: 17539638]

53. Newstadt JP, Mayo DJ, Inbaraj JJ, Subbaraman N, Lorigan GA. Determining the helical tilt of membrane peptides using electron paramagnetic resonance spectroscopy. J. Magn. Reson 2009;198:1-7. [PubMed: 19254856]

54. Lu JX, Blazyk J, Lorigan GA. Exploring membrane selectivity of the antimicrobial peptide KIGAKI using solid-state NMR spectroscopy. Biochim. Biophys. Acta - Biomembr 2006;1758:1303-1313.

55. Page RC, Lee S, Moore JD, Opella SJ, Cross TA. Backbone structure of a small helical integral membrane protein: A unique structural characterization. Protein Sci 2009;18:134-146. [PubMed: 19177358]

56. Respondek M, Madl T, Gobl C, Golser R, Zangger K. Mapping the orientation of helices in micellebound peptides by paramagnetic relaxation waves. J. Am. Chem. Soc 2007;129:5228-5234. [PubMed: 17397158]

57. Martinez-Seara H, Rog T, Pasenkiewicz-Gierula M, Vattulainen I, Karttunen M, Reigada R. Effect of double bond position on lipid bilayer properties: Insight through atomistic simulations. J. Phys. Chem. B 2007;111:11162-11168. [PubMed: 17760435]

58. Bunge A, Windeck A-K, Pomorski T, Schiller J, Herrmann A, Huster D, Muller P. Biophysical Characterization of a New Phospholipid Analogue with a Spin-Labeled Unsaturated Fatty Acyl Chain. Biophys. J 2009;96:1008-1015. [PubMed: 19186138]

59. Zanker PP, Jeschke G, Goldfarb D. Distance measurements between paramagnetic centers and a planar object by matrix Mims electron nuclear double resonance. J. Chem. Phys 2005;122:1-11. 024515.

60. Tiburu EK, Moton DM, Lorigan GA. Development of magnetically aligned phospholipid bilayers in mixtures of palmitoylstearoylphosphatidylcholine and dihexanoylphosphatidylcholine by solid-state NMR spectroscopy. Biochim. Biophys. Acta - Biomembr 2001;1512:206-214.

61. Wu F, Gaffney BJ. Dynamic behavior of fatty acid spin labels within a binding site of soybean lipoxygenase-1. Biochemistry 2006;45:12510-12518. [PubMed: 17029406]

62. Stillman AE, Schwartz LJ, Freed JH. Direct determination of rotational correlation time by electronspin echoes. J. Chem. Phys 1980;73:3502-3503. 


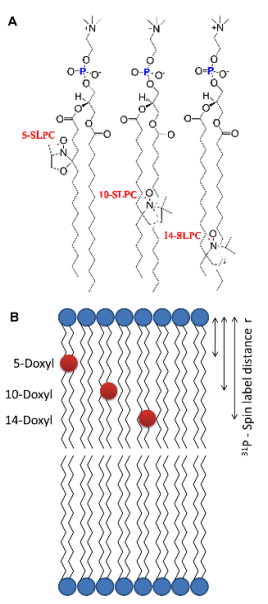

Fig. 1.

(A) Chemical structures of n-(4,4-dimethyl-oxazolidine-N-oxyl) -palmitoyl-2-stearoyl-snglycero-3-phosphocholine (n-DOXYL-PSPC, where $n=5,10,14)$ and $(B)$ the phospholipid bilayer model. The membrane immersion depth is defined as the shortest distance between the ${ }^{31} \mathrm{P}$ and the spin-label DOXYL. 


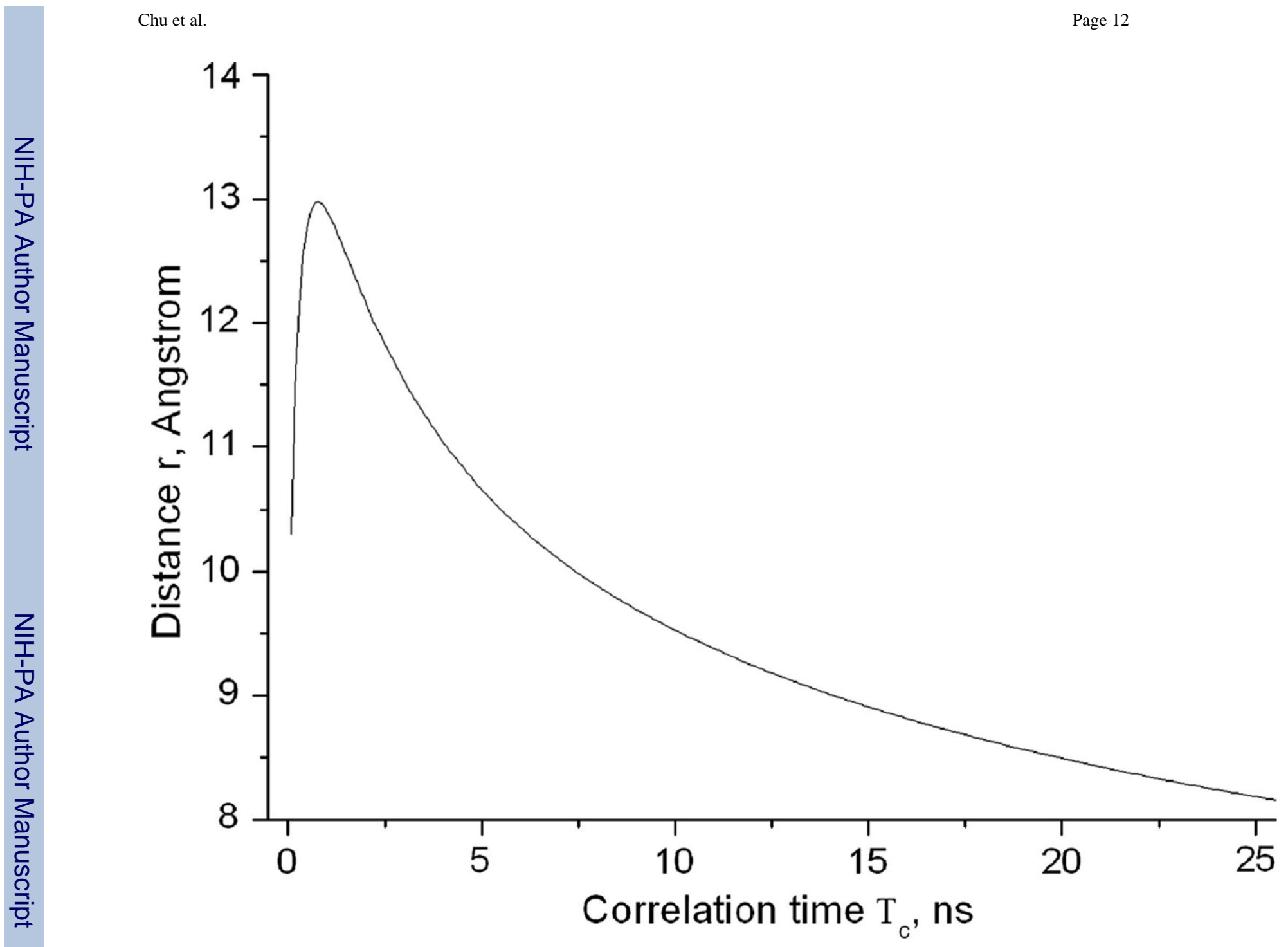

Fig. 2.

Spin label $-{ }^{31} \mathrm{P}$ distance $v s$. the correlation time $\tau_{\mathrm{c}}$. Calculated distance changes only in the range of 8 to $13 \AA$ whereas correlation time lies in the range from 0.1 to $25 \mathrm{~ns}$. 


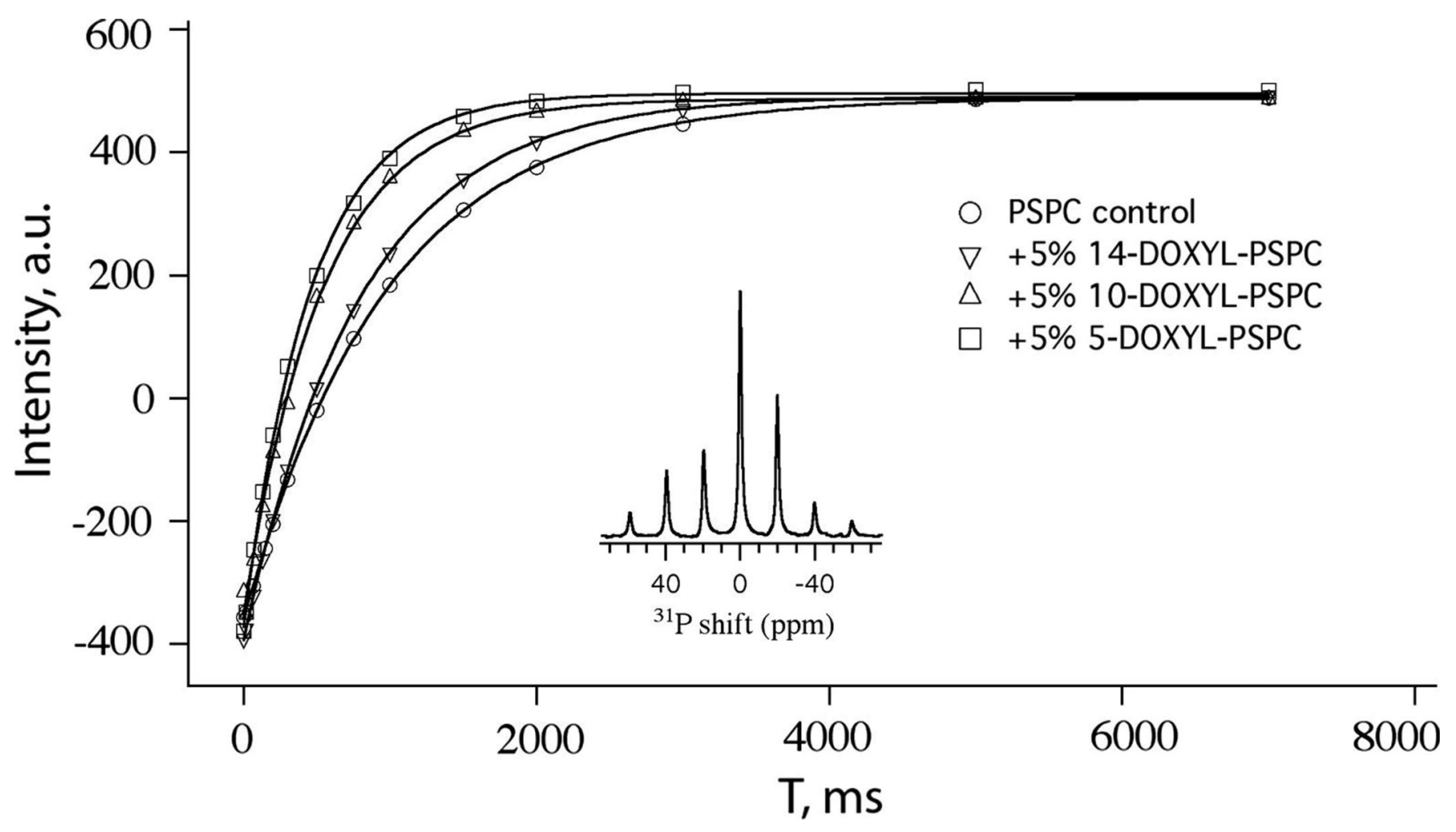

Fig. 3.

${ }^{31} \mathrm{P} \mathrm{T}_{1}$ vs. the position of DOXYL on PSPC at $5 \%$ (molar ratio) of spin-labels. The signal intensity profiles of ${ }^{31} \mathrm{P}$ are plotted as a function of the time delay in inversion recovery pulse sequence under control and in the presence of n-DOXYL PSPC. The insert is the typical ${ }^{31} \mathrm{P}$ MAS NMR spectrum used to measure ${ }^{31} \mathrm{P}_{1}$ by using inversion recovery, with sample spinning speed of $4 \mathrm{KHz}$ at the magic angle. 


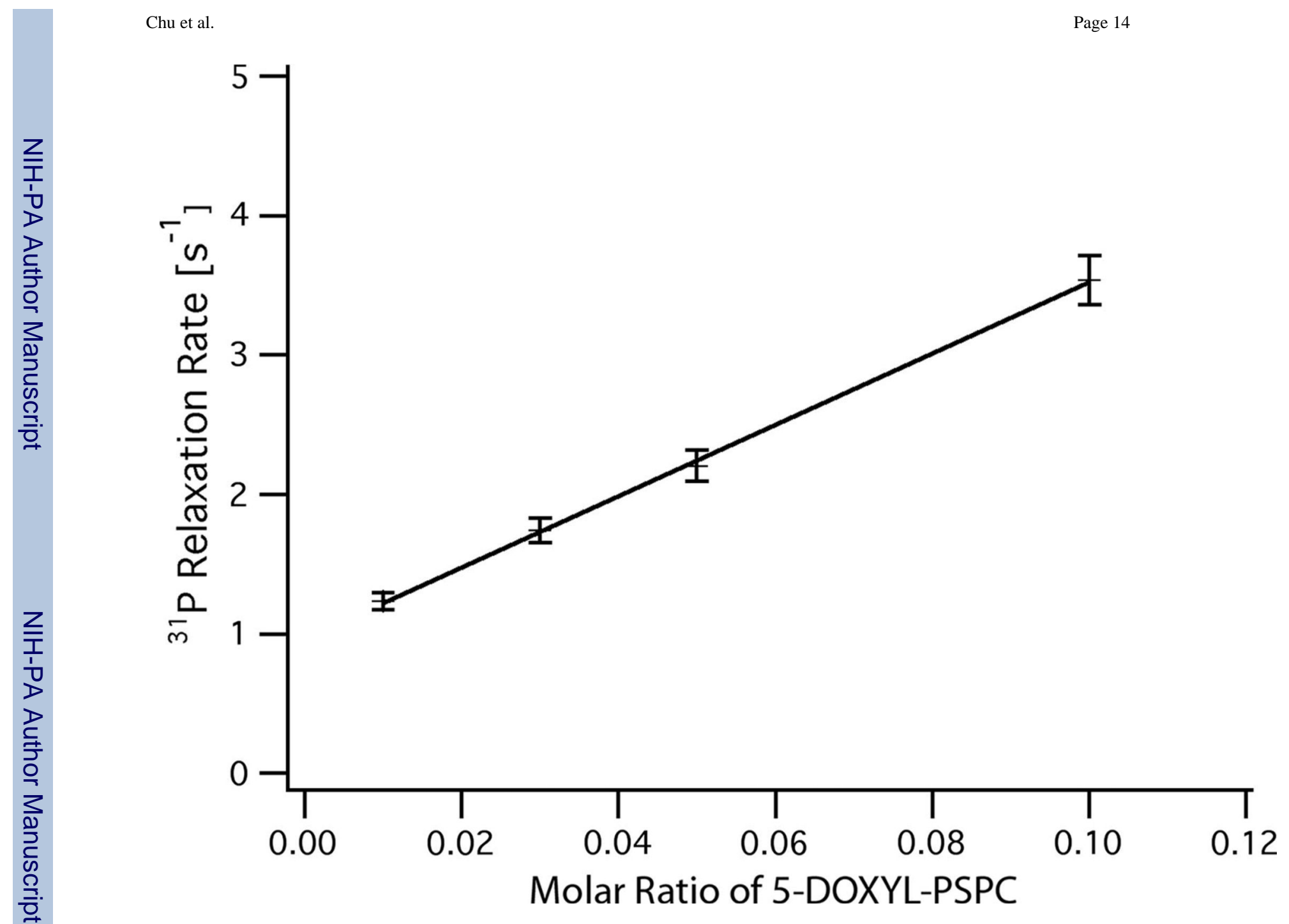

Fig. 4.

Plot of ${ }^{31} \mathrm{P} \mathrm{T}_{1}$ times $v s$. the concentration of 5-DOXYL-PSPC. A liner relationship (for details see text). 


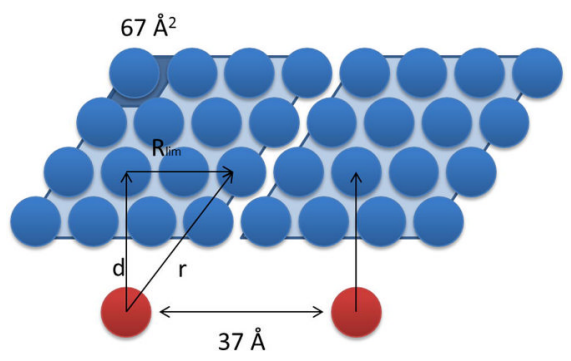

Fig. 5.

The concept model of the distance and the immersion depth (not drawn to scale). At a concentration of $5 \mathrm{~mol} \%$ spin labeled lipid, there is one spin label to relax nearby $20{ }^{31} \mathrm{P}$ nuclei. According to the area surface and the concentration, the distance between the observed ${ }^{31} \mathrm{P}$ and another nearby spin label would be at least $\sim 37 \AA$ away (see text). 


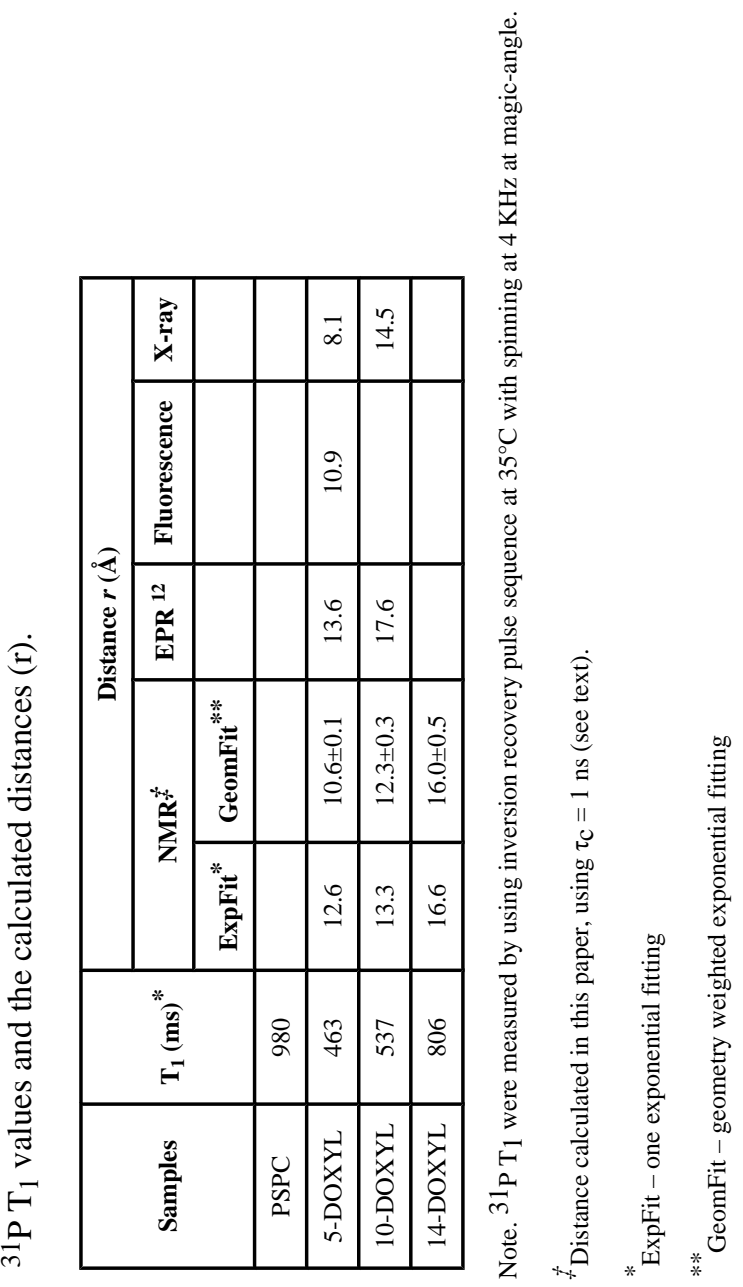

J Magn Reson. Author manuscript; available in PMC 2011 November 1. 